Research, Society and Development, v. 9, n. 3, e149932584, 2020

(CC BY 4.0) | ISSN 2525-3409 | DOI: http://dx.doi.org/10.33448/rsd-v9i3.2584

\title{
Como escrever uma dissertação de mestrado
}

How to write a master degree dissertation

Cómo escribir una disertación maestra

Recebido: 27/01/2020 | Revisado: 06/02/2020 | Aceito: 17/02/2020 | Publicado: 29/02/2020

Anna Carolina de Siqueira Ferreira

ORCID: https://orcid.org/0000-0003-2145-7301

Universidade Federal de Itajubá, Brasil

E-mail: anna@unifei.edu.br

Maria Gabriela Araújo Ranieri

ORCID: https://orcid.org/0000-0001-8631-020X

Universidade Federal de Itajubá, Brasil

E-mail: gabiranieri@unifei.edu.br

Patrícia Capellato

ORCID: https://orcid.org/0000-0002-6397-5820

Universidade Federal de Itajubá, Brasil

E-mail: pcapellato@gmail.com

Gilbert Silva

ORCID: https://orcid.org/0000-0002-3923-3982

Universidade Federal de Itajubá, Brasil

E-mail: gilbert@unifei.edu.br

Luiz Felipe Silva

ORCID: https://orcid.org/0000-0002-3174-9984

Universidade Federal de Itajubá, Brasil

E-mail: 1felipe@unifei.edu.br

\section{Resumo}

Esse trabalho é uma revisão bibliográfica de obras de vários autores sobre a escrita científica. Esse estudo sistemático foi desenvolvido com o objetivo de nortear os alunos durante o processo de elaboração de uma dissertação de mestrado com qualidade. Pois, A maioria dos programas de pós-graduação stricto sensu - no nível de mestrado - exige a escrita e entrega de uma dissertação para fins de conclusão do curso e obtenção do título de mestre. Nos últimos anos, muitos autores têm confirmado a baixa qualidade das dissertações de mestrado à 
nível mundial. Pois, escrever um texto acadêmico requer um conjunto de habilidades e competências do pesquisador. Assim, este artigo tem como objetivo apresentar um conjunto de diretrizes para a elaboração de uma boa dissertação de mestrado, através de análise bibliográfica de alguns dos artigos mais recentes sobre o assunto. Como resultado, é apresentada uma compilação das principais orientações voltadas à redação de dissertação de mestrado, tanto com relação à estrutura quanto ao seu conteúdo. Conclui-se que é importante e necessário fortalecer o preparo de alunos de mestrado para a escrita científica.

Palavras-chave: Dissertação de mestrado; Orientação; Qualidade.

\begin{abstract}
This work is a bibliographic review of works by various authors on scientific writing. The aim of this study was to develop a guiding to the student during the process of preparing a quality master's dissertation. Almost all stricto sensu postgraduate programs - at the master's degree require the writing and delivery of a dissertation in order to completion of the course and obtaining the master's degree in the specific area. However, the preparation of this report is not an easy task and it requires a set of the researcher's skills. In this context, many authors have confirmed the low quality of the dissertations, which is a concern worldwide. Thus, this article aims to present a set of guidelines for the elaboration of a good masters dissertation, through bibliographical analysis of the most recent articles about this theme. As a result, we presente a compilation of the main guidelines for dissertation writing, in terms of structure and content. We conclude that it is too important and necessary to consolidate the preparation of masters students for scientific writing.
\end{abstract}

Keywords: Guidelines; Master degree dissertation; Quality.

\title{
Resumen
}

Este trabajo es una revisión bibliográfica de trabajos de varios autores sobre escritura científica. Este estudio sistemático se desarrolló con el objetivo de guiar a los estudiantes durante el proceso de preparación de una disertación de maestría de calidad. La mayoría de los programas de posgrado stricto sensu, a nivel de maestría, requieren escribir y presentar una disertación con el fin de completar el curso y obtener el título de maestría. En los últimos años, muchos autores han confirmado la mala calidad de las disertaciones de maestría en todo el mundo. Para, escribir un texto académico requiere un conjunto de habilidades y competencias del investigador. Por lo tanto, este artículo tiene como objetivo presentar un conjunto de pautas para la preparación de una buena disertación de maestría, a través del 
análisis bibliográfico de algunos de los artículos más recientes sobre el tema. Como resultado, presentamos una compilación de las principales orientaciones para la escritura de tesis de maestría, tanto en términos de estructura como de contenido. Se concluye que es importante y necesario fortalecer la preparación de estudiantes de maestría para la escritura cientifica.

Palabras clave: Tesis de maestria; Orientaciones; Calidad.

\section{Introdução}

No âmbito dos programas de pós-graduação stricto sensu, a elaboração de uma dissertação de mestrado, baseada na condução de uma pesquisa científica, é requisito básico para a conclusão do curso e obtenção do título de mestre na área específica cursada. Trata-se de um trabalho de pesquisa realizado durante o curso de mestrado, que resultará em um relatório de caráter argumentativo, sobre um tema específico que deve ser intensivamente pesquisado, explorado e discutido (Komba, 2015).

Nesse sentido, o trabalho referente à dissertação de mestrado consiste na descoberta de algo, ainda que seja a título de confirmação do conhecimento já existente contribuindo igualmente para o desenvolvimento da ciência. Espera-se, com a dissertação, que o futuro mestre desenvolva competências para avaliar os resultados de outras pesquisas, descobrir as coisas por si mesmas, bem como se comunicar de forma eficaz (Parsons \& Knight, 2015).

Através da redação do relatório da pesquisa de mestrado - a dissertação, o pesquisador deverá demonstrar domínio da área temática pesquisada (Komba, 2015), ou seja, mostrar que conhece bem o assunto sobre o qual está desenvolvendo sua pesquisa.

Embora seja requisito comum entre os programas de pós-graduação a nível de mestrado, a redação de dissertações não é tarefa fácil, em virtude de sua complexidade e desafios enfrentados na confecção deste relatório de pesquisa (Attard, 2018; Komba, 2015). A baixa qualidade desses textos é confirmada por diversos estudos e tem sido objeto de preocupação a nível mundial (Komba, 2015).

Desse modo, este artigo tem como objetivo apresentar diretrizes sobre como escrever uma boa dissertação de mestrado, com base em uma análise bibliográfica de alguns dos artigos mais recentes sobre o assunto. Contemplando tanto questões voltadas ao 
conteúdo quanto à estrutura desse trabalho acadêmico-científico, espera- se favorecer a melhoria da qualidade das produções.

Justifica-se a importância desta proposta, tendo em vista a relevância da pesquisa científica no desenvolvimento do conhecimento e, especialmente, a necessidade de comunicação das informações pesquisadas de forma eficaz.

Em função da complexidade em se elaborar uma dissertação de mestrado de qualidade, há alguns estudos voltados a auxiliar na preparação desse trabalho acadêmico, seja através de experiência individual (Kohl, 2016), baseado em normativas (Takahashi et al., 2017) ou com enfoque no projeto de pesquisa como processo anterior à escrita da dissertação propriamente dita (Attard, 2018).

Por sua vez, outros trabalhos direcionam atenção maior aos desafios encontrados no processo de redação das dissertações, com fins de conhecer os problemas (Komba, 2015; Ramalho, Garcia \& Carneiro, 2018) e tentar solucioná-los ou minimizá-los (Komba, 2015).

Enfim, neste artigo serão consideradas todas as contribuições dos trabalhos selecionados na revisão bibliográfica, para fins de compilação e apresentação das principais diretrizes para a elaboração de uma dissertação de mestrado de qualidade.

\section{Metodologia}

Este trabalho foi desenvolvido durante a disciplina "Comunicação Científica" oferecida pelo programa de pós-graduação em Desenvolvimento, Tecnologia e Sociedade do Instituto de Engenharia de Produção da Universidade Federal de Itajubá, campus de Itajubá. Trata-se de uma revisão bibliográfica onde são consideradas as contribuições de outros autores sobre a escrita científica em publicações entre os anos 2015 - 2019. Esse estudo sistemático tem como objetivo contribuir com o processo de elaboração de textos científicos a fim de nortear os alunos de pós-graduação a desenvolverem seus textos acadêmicos.

A estrutura deste trabalho está dividida em quatro seções, além da introdução, temos a segunda que é a metodologia, na terceira apresenta-se a fundamentação teórica sobre o assunto e a quarta seção apresenta os resultados do estudo.

\section{Fundamentação Teórica}


Research, Society and Development, v. 9, n. 3, e149932584, 2020

(CC BY 4.0) | ISSN 2525-3409 | DOI: http://dx.doi.org/10.33448/rsd-v9i3.2584

\subsection{A dissertação de Mestrado}

No âmbito acadêmico do mestrado, após a escolha do tema e a condução da pesquisa científica, procede-se ao relato da investigação proposta e realizada. Este relato consiste na dissertação propriamente dita, com estrutura definida pela Associação Brasileira de Normas Técnicas (ABNT), responsável por padronizar as técnicas de produção feitas no país (Takahashi et al., 2017).

Conceitualmente, a dissertação de mestrado pode ser considerada como "resultado de um trabalho experimental ou exposição de um estudo científico retrospectivo, de tema único e bem delimitado em sua extensão, com o objetivo de reunir, analisar e interpretar informações" (Ibdem, 2017).

Em outras palavras, a dissertação é um relatório extenso, de caráter predominantemente argumentativo, baseado na leitura e análise profundas da literatura sobre um tópico específico, através de um diálogo coerente, coeso e lógico (Ramalho, Garcia \& Carneiro, 2018; Vos \& Armstrong, 2019).

Além das características que uma dissertação deve possuir, é imprescindível que o pesquisador desenvolva e integre algumas habilidades suas como: autogestão, determinação, objetividade, adaptabilidade e reflexão (Vos \& Armstrong, 2019).

Como todo trabalho científico, a dissertação também deve respeitar o quesito de originalidade, ou seja, a não repetição de um trabalho já realizado: ou desenvolvendo algo inovador ou adotando uma nova abordagem sobre o tema proposto (Parsons \& Knight, 2015). Por isso é necessária a realização de uma revisão abrangente e adequada da literatura, a fim de evitar o plágio.

De modo geral, pode-se constatar a complexidade da redação deste trabalho final de mestrado, que exige treino e uso de técnicas adequadas (Ramalho, Garcia \& Carneiro, 2018). Apesar disso, a escrita de uma dissertação traz benefícios ao pesquisador, listados na Figura 1 a seguir: 


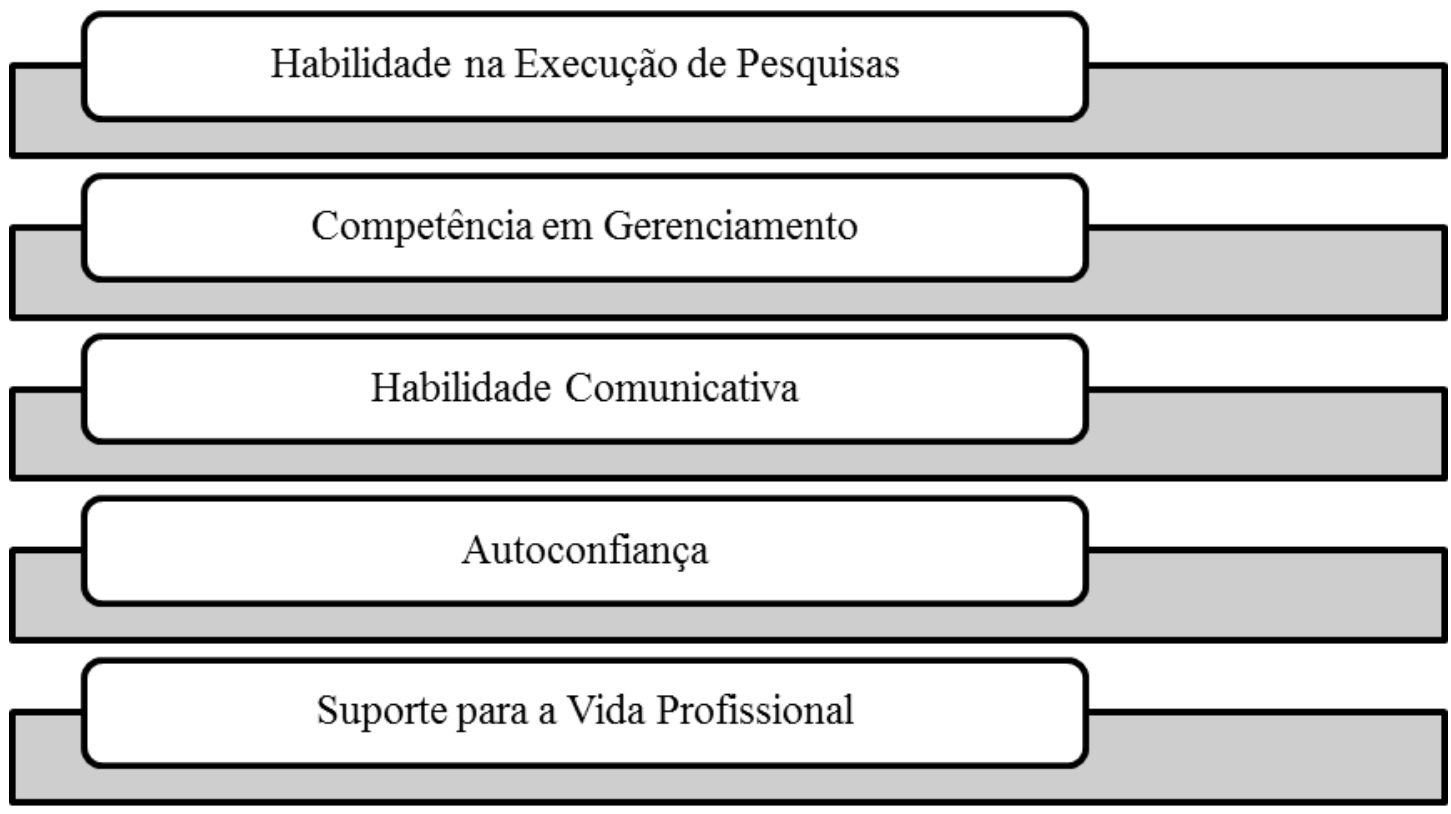

Figura 1. Benefícios ao escrever uma dissertação de mestrado. Fonte: (Parsons \& Knight, 2015).

A Figura 1 apresenta algumas habilidades que o pesquisador pode adquirir ou desenvolver durante o mestrado. Pois, o nível de ensino de aprofundamento é superior na pós-graduação stricto sensu - em relação aos cursos de especialização.

\subsection{Antes da escrita}

Antes de se iniciar a redação do trabalho científico, é necessário planejamento adequado, através de muita leitura e trabalho organizacional, incluindo a gestão do tempo. Ainda que nada disso apareça no relatório final, é essencial dedicação e persistência (Kohl, 2016; Parsons \& Knight, 2015).

No que diz respeito ao planejamento, tanto para a condução da pesquisa quanto para o seu relato, é preciso delinear um plano de ação, ou seja, um roteiro do que se pretende fazer (Attard, 2018).

Cabe salientar que, como todo trabalho científico, a dissertação de mestrado deve apresentar linguagem adequada. Elementos como clareza, concisão e objetividade, além do respeito às normas gramaticais, são imprescindíveis para uma boa escrita científica e devem ser de competência do pesquisador (Ramalho, Garcia \& Carneiro, 2018). 
Research, Society and Development, v. 9, n. 3, e149932584, 2020

(CC BY 4.0) | ISSN 2525-3409 | DOI: http://dx.doi.org/10.33448/rsd-v9i3.2584

Conciliar estudo e vida pessoal nem sempre é algo fácil, então até o tempo que o indivíduo terá para dedicar-se aos estudos fora da sala de aula, deve ser considerado no planejamento. Com tudo planejado adquadamente a pesquisa torna-se mais eficente e prazerosa.

\subsection{Desafios}

Alguns desafios referentes à elaboração de dissertações de mestrado têm sido apontados por diversos autores, como é o caso de Komba (2015). O autor procurou investigar tais desafios, através da análise de 39 teses e 64 dissertações de três diferentes universidades da Tanzânia. Como resultado, o estudo confirmou inúmeros desafios na redação, incluindo falta de compreensão adequada da função de algumas seções (Komba, 2015).

No Brasil, o estudo de Ramalho, Garcia \& Carneiro (2018), por exemplo, também procurou identificar os problemas técnicos e linguísticos em dissertações na área de Educação, através da análise de 10 dissertações de diferentes instituições de ensino superior. Como resultado, todas elas apresentaram algum problema: seja na estrutura, na forma ou no conteúdo (Ibidem, 2018). A Figura 2 a seguir apresenta de forma resumida os principais desafios encontrados em dissertações de mestrado: 


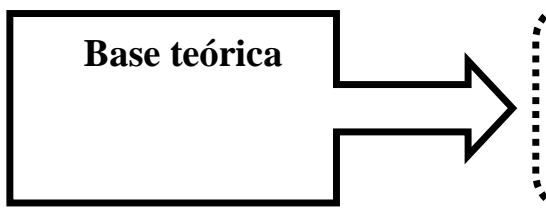

Sem justificativa para realizar o estudo;

Sem explicação sobre o conhecido/desconhecido sobre o tema.

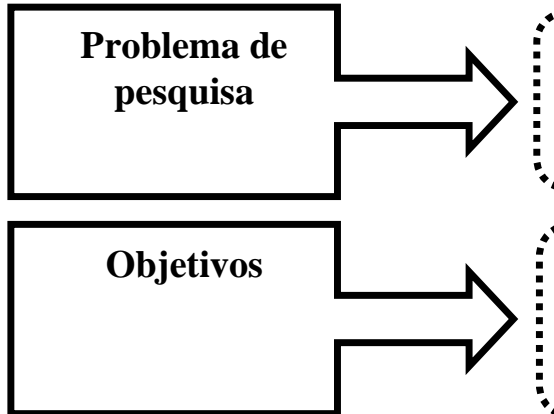

Sem clareza e relação com o título;

Extensos.

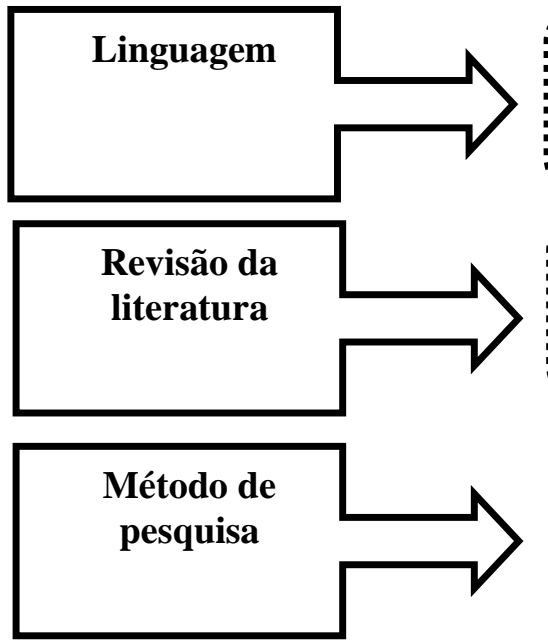

Erros diversos (gramaticais estilísticos, etc.).

Duplicados;

Redundantes.<smiles>C1CCCCC1</smiles>

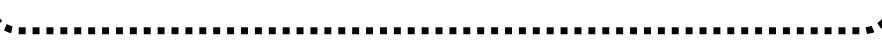

Sem viés crítico ou identificação de lacunas;

Pontos fracos em fazer citações.

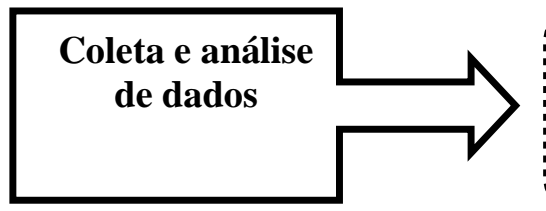

Sem justificativa para seleção do método ou do objeto de pesquisa;

Sem clareza sobre os procedimentos
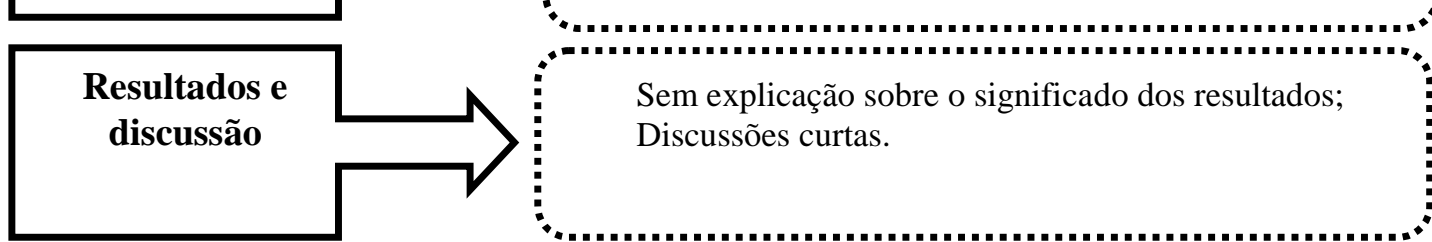

Sem justificativa para seleção das ferramentas

Sem menção às ferramentas usadas na análise quantitativa

Sem explicação sobre o significado dos resultados;

Discussões curtas.

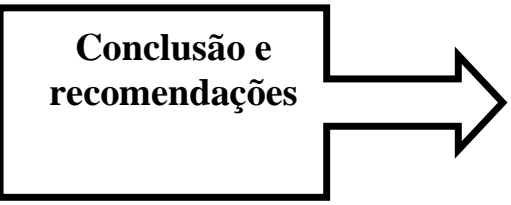

Confusão entre conclusão e resumo;

Recomendações apresentadas de forma inadequada.

Figura 2: Principais desafios encontrados em dissertações de mestrado. Fonte: Komba (2015).

Na Figura 2, é possível observar onde estão as maiores dificuldades encontradas nas dissertações de mestrado. 
Research, Society and Development, v. 9, n. 3, e149932584, 2020

(CC BY 4.0) | ISSN 2525-3409 | DOI: http://dx.doi.org/10.33448/rsd-v9i3.2584

Com base nas considerações apresentadas na seção anterior (2.2), esse artigo tem por objetivo orientar o processo de escrita de uma dissertação de mestrado, o que acaba contribuindo para a comunicação científica no geral.

Quanto aos procedimentos, esta se caracteriza como uma pesquisa bibliográfica, uma vez que "os dados provêm apenas ou prioritariamente das referências teóricas" (Nascimento, 2016).

Nesse contexto, foram realizadas buscas em diversas bases de dados, como Scopus, Science Direct, Google Acadêmico, Scielo e CAPES Periódico, cujo filtro de pesquisa foi: artigos publicados no período de 2015 a 2019 sobre como escrever uma dissertação de mestrado.

O próximo passo foi selecionar os artigos mais relevantes, através da leitura e análise de seus respectivos resumos, verificando a relação entre o assunto abordado em cada estudo e o escopo deste trabalho.

Deste modo, após análise crítica dos artigos selecionados, foram sintetizadas as principais orientações sobre como elaborar uma dissertação de mestrado de qualidade, com vistas aos requisitos básicos de escrita científica.

\section{RESULTADOS}

Após leitura e análise dos artigos selecionados, que compõem a seção de Referências deste trabalho, foram compiladas as orientações sobre como elaborar uma dissertação de mestrado de qualidade.

Cabe ressaltar que, como resultado de uma pesquisa científica bem estruturada, a dissertação de mestrado deve ser relatada de maneira clara e lógica, a fim de propiciar a compreensão e conhecimento do leitor e facilitar a replicação do que foi feito (Parsons \& Knight, 2015). Para isso, o pesquisador deve escrever com clareza e apresentar capacidade de raciocínio, análise e síntese (Parsons \& Knight, 2015).

Nesta seção, primeiramente, apresenta-se a estrutura ordenada de uma dissertação de mestrado, que deve conter seções de texto claramente identificáveis (Parsons \& Knight, 2015). Em seguida, cada seção de texto será descrita quanto ao seu respectivo conteúdo. 


\subsection{Estrutura}

A estrutura de uma dissertação de mestrado é dividida em três partes: pré- texto, texto e pós-texto (Takahashi et al., 2017). Cada uma dessas partes da dissertação possui determinadas seções, conforme Figura 3 a seguir:

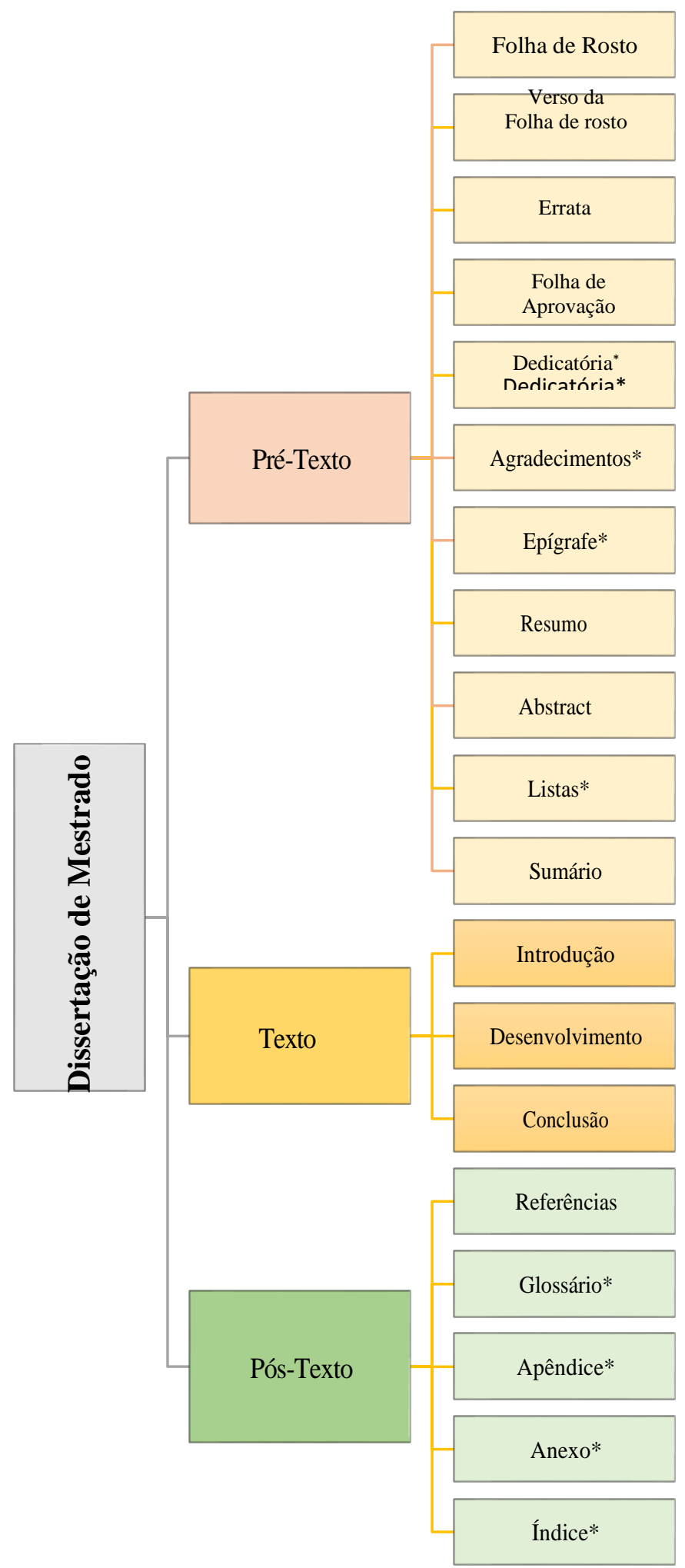

Figura 3: Estrutura de uma dissertação. Fonte: Elaborado pela autora (Takahashi et al., 2017). 
Research, Society and Development, v. 9, n. 3, e149932584, 2020

(CC BY 4.0) | ISSN 2525-3409 | DOI: http://dx.doi.org/10.33448/rsd-v9i3.2584

Analisando a Figura 3, é possível observar com mais clareza a divisão da estrutura de uma dissertação, isso poderá facilitar na hora de iniciar o processo de escrita.

Os itens apresentados no terceiro nível do organograma acima (Figura 3) estão listados de acordo com a ordem em que devem ser apresentados no trabalho de dissertação de mestrado. Ressalta-se que o uso dos itens com (*) é facultativo.

\subsection{Conteúdo das seções}

Embora o título de uma dissertação de mestrado não seja uma seção do trabalho, ele é imprescindível. Seu objetivo é identificar a questão de pesquisa, definindo o escopo do estudo de forma clara, concisa e interessante (Attard, 2018). Ou seja, o título deve ser relacionado ao que se propõe a pesquisa.

A seguir, são apresentadas as seções de texto de uma dissertação. Cabe enfatizar que os elementos pré e pós-textuais, com exceção do resumo/abstract, não serão ilustrados ou descritos neste artigo. Para conhecê-los, pode-se consultar o trabalho de Takahashi et al. (2017).

\subsubsection{Resumo}

O resumo de uma dissertação de mestrado deve conter, no máximo, de 150 a 500 palavras e ser redigido em um único parágrafo. Além disso, devem ser usadas frases concisas, afirmativas, na voz ativa e na terceira pessoa do singular. As palavras-chave devem ser separadas por ponto (Takahashi et al., 2017).

Quanto ao seu conteúdo, o resumo precisa dar ênfase ao objetivo, método de pesquisa, resultados obtidos e conclusões do estudo.

\subsubsection{Introdução}

A introdução é a seção inicial do texto, que deve conter a delimitação do assunto tratado, os objetivos do estudo e outros elementos necessários para situar e justificar a proposta do trabalho (Takahashi et al., 2017). Ou seja, é preciso mostrar a relevância em se pesquisar o que se propõe.

$\mathrm{Na}$ introdução, devem ser apresentados os antecedentes do problema de pesquisa, como: histórico teórico, problema de pesquisa, objetivos específicos e justificativos da 
Research, Society and Development, v. 9, n. 3, e149932584, 2020

(CC BY 4.0) | ISSN 2525-3409 | DOI: http://dx.doi.org/10.33448/rsd-v9i3.2584

proposta (Attard, 2018; Komba, 2015). Nesse sentido, o problema de pesquisa deve ser relevante em seu campo de estudo (Parsons \& Knight, 2015).

Ao contextualizar o tema, é importante explicar o que motivou a pesquisa e como ela se encaixa no conhecimento já existente. Dessa forma, o pesquisador precisa conhecer o que foi feito antes, o que está sendo feito e o que precisa ser feito a seguir. Tudo isso demonstra o nível de experiência do autor (Parsons \& Knight, 2015).

\subsubsection{Fundamentação Teórica ou Revisão de Literatura}

Após a seção de introdução, há a seção de revisão de literatura, também chamada de fundamentação teórica ou revisão bibliográfica. Independente de nomenclaturas, esta seção deve apresentar revisões críticas da literatura sobre o tema estudado, usando conhecimentos produzidos no passado como base para análise e discussão (Komba, 2015; Ramalho, Garcia \& Carneiro, 2018).

O pesquisador sempre deve se respaldar em estudos anteriores e utilizá-los como arcabouço teórico para seu próprio trabalho. Em outras palavras, deve-se relacionar seu tema com a literatura de pesquisa existente e mostrar que está familiarizado com ela. $\mathrm{O}$ intuito é convencer o leitor a querer ler mais (Parsons \& Knight, 2015).

Deve-se buscar na literatura evidências convincentes de que existedemanda para o estudo proposto (Attard, 2018), ou seja, justificar a importância em se pesquisar o que se pretende.

Além do suporte teórico, a revisão bibliográfica é extremamente relevante para o desenvolvimento da pesquisa, pois ajuda a delimitar seus objetivos e dar consistência à dissertação (Kohl, 2016).

Um dos desafios relacionados à esta seção é a ausência de análise crítica da literatura. Muitas vezes, as dissertações apresentam apenas um resumo dos trabalhos existentes, mas não os analisa adequadamente (Komba, 2015). Assim, através de uma boa revisão bibliográfica, é possível identificar o conhecido e também as lacunas a serem exploradas (Attard, 2018).

\subsubsection{Metodologia ou Materiais e Métodos}

Esta seção, ora intitulada como Metodologia, ora como Materiais e Métodos é voltada a relatar como se deu a resolução do problema de pesquisa apresentado no início do trabalho. Para tanto, segundo Parsons \& Knight (2015), o pesquisador deve pensar e 
Research, Society and Development, v. 9, n. 3, e149932584, 2020

(CC BY 4.0) | ISSN 2525-3409 | DOI: http://dx.doi.org/10.33448/rsd-v9i3.2584

escolher uma abordagem metodológica que permita tratar e, também, responder sua pergunta (Parsons \& Knight, 2015).

Esta parte da dissertação deve conter os procedimentos e ferramentas de geração e análise de dados, além de justificativa tanto para a escolha do método e instrumentos, quanto do objeto de pesquisa (Komba, 2015).

Toda pesquisa deve ter métodos claramente definidos em todas as etapas, como medição, coleta e análise de dados. É imperativo que o pesquisador desenvolva corretamente seu trabalho e descreva com precisão e detalhamento o que e como foi feito (Parsons \& Knight, 2015).

Antes mesmo da aplicação prática da proposta, deve-se pensar sobre o tipo e quantidade de dados a serem coletados, e também sobre o que fazer com eles depois da coleta (Parsons \& Knight, 2015).

Todas as seções de uma dissertação, assim como a dissertação em si, devem ser planejadas cuidadosamente. E isso não é diferente no caso da metodologia. É preciso planejar e analisar qual a melhor estratégia e número de amostragem, o melhor método e/ou instrumento para coleta e análise de dados. Tudo isso depende da questão de pesquisa.

Todos esses elementos são de vital importância para validar os resultados da pesquisa e permitir sua aplicabilidade (Attard, 2018).

\subsubsection{Resultados}

A seção de resultados de uma dissertação consiste em apresentar, analisar, interpretar e discutir os dados coletados, de acordo com os objetivos da pesquisa ou hipóteses anteriormente definidas (Komba, 2015). Ou seja, é importante relacionar os resultados com os objetivos iniciais do estudo e interpretar essa relação.

Ainda nesta seção, é indispensável que o pesquisador realize a discussão dos resultados e não se restrinja a apenas comentá-los. A discussão das descobertas é crucial, pois é onde reside a contribuição intelectual do autor como pesquisador (Komba, 2015).

\subsubsection{Conclusão}

Esta seção contempla o resumo do trabalho, as conclusões resultantes da pesquisa e recomendações para outros estudos complementares (Komba, 2015). Em suma, é um 
capítulo breve que reafirma o significado da proposta e enfatiza a razão pela qual foi relevante investigar o problema (Attard, 2018).

A seção de Conclusão tem por objetivo ir além da resposta básica à pergunta de pesquisa, vinculando as próprias descobertas com as ideias de outros pesquisadores. Em outras palavras, trata-se de resumir as descobertas e inseri-las em um contexto intelectual mais amplo, onde a dissertação foi inicialmente definida (Parsons \& Knight, 2015).

As conclusões devem corresponder aos objetivos propostos, fazendo referência ao capítulo introdutório e à literatura existente sobre o assunto. $\mathrm{O}$ autor deve demonstrar como o novo conhecimento se encaixa, contradiz, ou apoia a literatura anterior (Parsons \& Knight, 2015).

Além disso, é a seção da dissertação onde sugerem-se outras linhas de pesquisa ou abordagens alternativas (Parsons \& Knight, 2015).

Deve-se ter sempre em mente de que a conclusão é uma das partes mais importantes de um texto científico, pois é o momento de finalizar o que foi desenvolvido no conteúdo e reforçar a ideia principal.

\section{Considerações finais}

Este trabalho tem o intuito de contribuir na hora de iniciar a escrita de uma dissertação de mestrado. Pois, em sua grande maioria, os programas de pós-graduação stricto sensu nível de mestrado - exigem como requisito a escrita de um relatório baseado em pesquisa científica, para fins de conclusão do curso e obtenção do título de mestre na área específica.

Foram apresentadas as principais orientações de especialistas na área da comunicação científica dos últimos anos e assim, espera-se tornar a jornada acadêmica mais agradável e eficiente.

A elaboração deste relatório não é tarefa fácil e requer um conjunto de habilidades e competências do pesquisador, como o domínio da área temática, conhecimento linguístico e textual, autogestão, organização, dentre outros.

Muitos autores têm confirmado a baixa qualidade das dissertações, objeto de preocupação a nível mundial. Nesse sentido, este artigo procurou apresentar um conjunto 
Research, Society and Development, v. 9, n. 3, e149932584, 2020

(CC BY 4.0) | ISSN 2525-3409 | DOI: http://dx.doi.org/10.33448/rsd-v9i3.2584

de orientações para a elaboração de uma boa dissertação de mestrado, com base na análise bibliográfica de alguns dos artigos mais recentes sobre o assunto.

A título de ilustração e resumo, são apresentadas as principais qualidades de uma dissertação na Figura 4 a seguir:

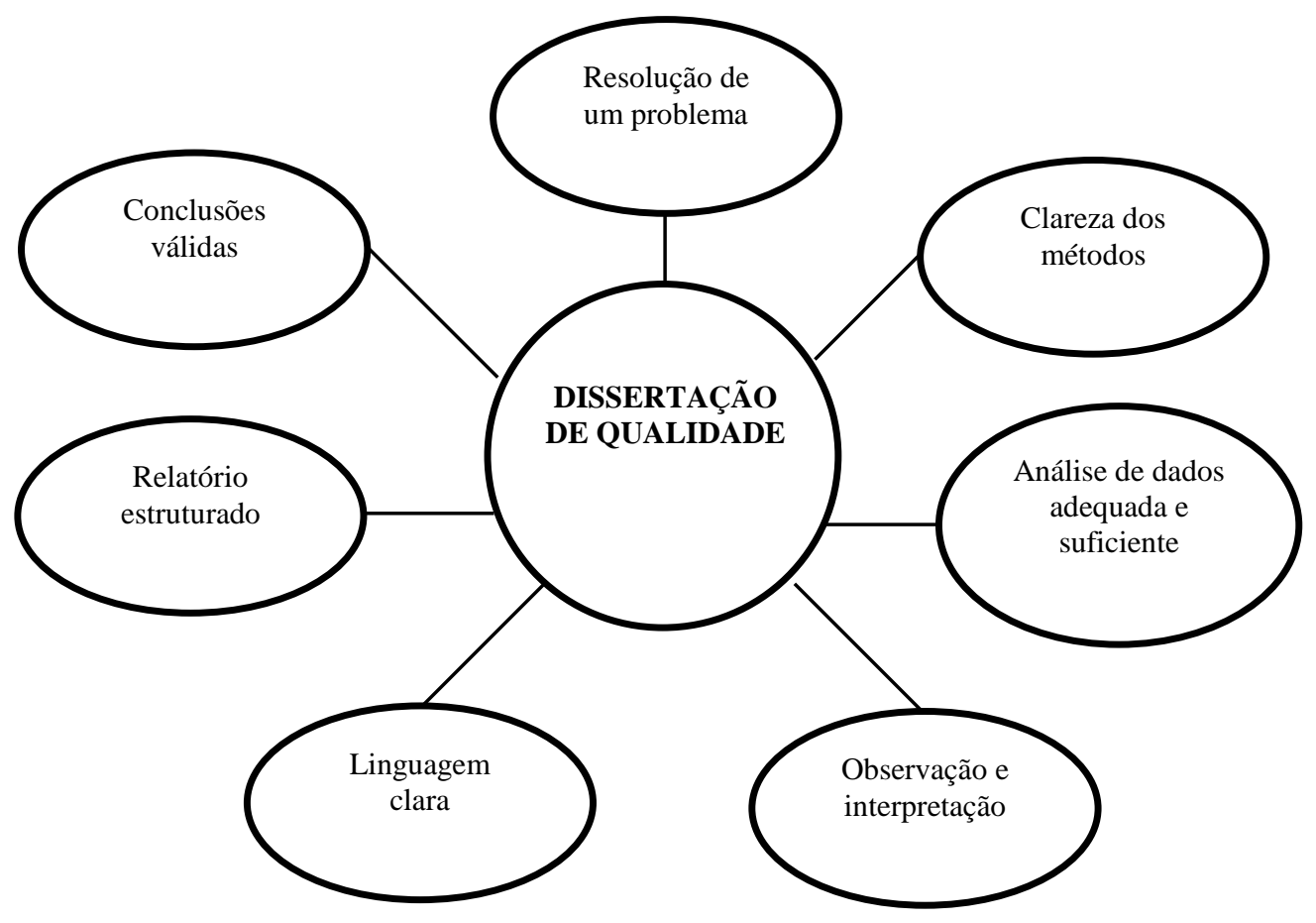

Figura 4: Características principais de uma boa dissertação. Fonte: Elaborado pela autora (Parsons \& Knight, 2015).

De acordo com Vos \& Armstrong (2019), a forma como os métodos de pesquisa estão sendo ensinados não é ideal para preparar os alunos para a dissertação. Nesse sentido, recomenda-se a revisão e reformulação dos cursos de metodologia oferecidos pelas universidades, com foco especial na escrita de relatórios de pesquisa (Komba, 2015). Portanto, acredita-se que seja importante para os programas de pós-graduação, oferecer disciplinas para dar suporte e ajudar a preparar os alunos de mestrado para a escrita científica.

\section{Referências}


Attard, N. (2018). WASP (Write a Scientific Paper): Writing an academic research proposal. Early Human Development, 123(1): 39-41.

Kohl, K. (2016). O prelúdio da escrita de uma dissertação. Palíndromo, 8(15), 89-96.

Komba, S. C. (2015). Chalenges of writing theses and dissertations among postgraduate students in Tanzanian higher learning institutions. International Journal of Research Studies in Education, 5(3), 71-80.

Nascimento, F. P. (2016). Metodologia da Pesquisa Científica: Teoria e Prática (1a ed.). Brasília: Thesaurus.

Parsons, T., Knight, P. (2015). How To Do Your Dissertation in Geography and Related Disciplines. London: Routledge, https://doi.org/10.4324/9781315849218.

Pereira, A.S. et al. (2018). Metodologia da pesquisa científica. [e-book]. Santa Maria. Ed. UAB/NTE/UFSM. Disponível em: https://repositorio.ufsm.br/bitstream/handle/1/15824/Lic_Computacao_MetodologiaPesquisa-Cientifica.pdf?sequence=1. Acesso em: 05 fev. 2020.

Ramalho, R. de O.; Garcia, F. J. J. P. \& Carneiro, J. A. V. (2018). Os desafios da escrita científica: uma análise dos capítulos teóricos de 10 Dissertações de Mestrado em Educação. Evidência: olhares e pesquisa em saberes educacionais, 14(15), 111-124.

Takahashi, J. A.; Filet, N. B.; Gardim, S. M. \& Saheki, Y. (2017). Guia prático para elaboração de dissertação, tese, monografia e projeto de pesquisa. Universidade de São Paulo. Escola de Enfermagem. Biblioteca "Wanda de Aguiar Horta". Recuperado em: 14 fevereiro, 2020 , http://:www.ee.usp.br/biblioteca/doc/Manual2017.pdf+\&cd=15\&hl=pt-

$\mathrm{BR} \& \mathrm{ct}=\mathrm{clnk} \& \mathrm{gl}=\mathrm{br}$

Vos, L. \& Armstrong, K. (2019). Context and process challenges associated with supervising postgraduate dissertation: An example from marketing. International Journal of Management Education, 17(1), 47-61. 


\section{Porcentagem de contribuição de cada autor no manuscrito}

Anna Carolina de Siqueira Ferreira - 35\%

Maria Gabriela Araújo Ranieri - 30\%

Patrícia Capellato - 25\%

Gilbert Silva $-5 \%$

Luiz Felipe Silva - 5\% 